\title{
Modelling of the Employment Distribution in the System of Regional Enterprises in Digital Economy
}

\author{
Azarnova T.V.* \\ Voronezh State University \\ Voronezh, Russia \\ e-mail: ivdas92@mail.ru \\ Kashirina I.L. \\ Voronezh State University \\ Voronezh, Russia \\ e-mail: kash.irina@mail.ru
}

\author{
Bondarenko Yu.V. \\ Voronezh State University \\ Voronezh, Russia \\ e-mail: bond.julia@mail.ru \\ Polovinkina M.V. \\ Voronezh State University of Engineering Technologies \\ Voronezh, Russia \\ e-mail: polovinkin@yandex.ru
}

\begin{abstract}
The article studies mathematical models based on the kinetic equation of transfer. We apply these models to assess the dynamics of the employment distribution in the system of small and medium-sized regional enterprises in the digital economy, which is characterized by a shift of entrepreneurship towards high-tech industries. Solving innovative problems and focusing on the introduction of digital technologies, the small and medium-sized enterprises are the most important driver of the digital economy. In the digital economy, organizational changes will inevitably occur in the structure of the economic complex of any region. The study of the projected dynamics plays an important role for efficient management policies aimed at regulating the balanced GRP growth of small and medium-sized enterprises and effective employment. The management policy of supporting small and medium-sized businesses can be implemented through the formation of certain factors that affect the functions of the life cycle, the creation and closure of objects included in it. The paper uses a population-based approach to study the impact of the life cycle function, creation, and closure of entities on the distribution of employment within the system of small and medium-sized enterprises. Differential equations are considered for the case of a stationary and non-stationary nature of the dependence of the growth functions, creation and closure of entities on time. Numerical algorithms are proposed for solving the analyzed differential equations and finding the employment distribution in the system of regional enterprises.
\end{abstract}

Keywords - digital economy, mathematical models of population dynamics.

\section{INTRODUCTION}

In digital economy system, much attention is paid to the development of small and medium enterprises [1,2]. Small enterprises can quickly adapt to the conditions of dynamic changes taking place in the era of the digital economy. The digital economy is carried out with the help of digital telecommunications, and this is a favorable factor for the development of small enterprises. New digital business models are aimed at reducing costs and optimizing the interaction of business entities. In the digital economy, small business is directly related to improving the quality of the economic space in terms of its density, location and connectivity.

This paper considers tools based on the population approach presented in the form of models and algorithms which allow obtaining estimates of the employment distribution dynamics in the system of small and mediumsized enterprises. The population approach is an effective way to study systems of objects that have common patterns in their development dynamics. The tools obtained in this work allow investigating the structure of employment and using its results as means of supporting decision-making in developing a system of state support for business in digital economy.

\section{LITERATURE REVIEW}

Many Russian and foreign researchers studied the dynamics of organizational changes in the system of enterprises in a region or industry in terms of their number, size distribution, distribution of total assets and profits: R. Gibrat, T. Bates, D. B. Audretsch, L. Samuelson, R. Cressy, G. Becker, M. Robertson, D. Evans, D. Holts-Ikin, V.M. Polterovich, G. B. Kleiner, V.L. Makarova, Z. Coase, A. Shastitko, T. Dunne, B. Zhovanovik, P. Geroski, J. Mat, P. Portugal, R. Shmalenzi, D. MacFadden, V. Green, J. Heckman, P. Rudh, T. Lancaster, J. Zheveke, M. Kine, V. Hajivasiliu, A.T. Mustafin, A.Yu. Kantarbayeva, P.G. Aleksashin, V. Yu. Belousova, E.S. Popova, Yu.S. Pinkovetskaya. A fairly well-known work in the field of studying the dynamics of the distribution of firms by size is an article by Robert Gibrat [3]. In this work, Robert Gibrat shows that if increments of the firm size are identically distributed independent random variables then the firm size distribution is coming to obey the lognormal distribution law. The established fact is called the law of proportional growth (Gibrat's Law). The law is based on three assumptions: the growth rate of all firms over a certain period does not depend on its size; growth rate does not depend on the individual characteristics of the company; the firm's growth rates in two consecutive periods are independent values. When these 
assumptions are fulfilled, the size increment of the company is proportional to its current achieved size:

$$
\begin{gathered}
x(t)-x(t-1)=\varepsilon(t) x(t-1), \\
x(t)=x(0)(1+\varepsilon(1))(1+\varepsilon(2)) \ldots(1+\varepsilon(t)),
\end{gathered}
$$

$\log x(t)=\log x(0)+\log (1+\varepsilon(1))+\log (1+\varepsilon(2))+\ldots+\log (1+\varepsilon(t))$, where $\varepsilon(t)$ is normally distributed random variable with parameters $\mu, \sigma^{2}$. If $t \rightarrow \infty$, the distribution $\log x(t)$ tends asymptotically to the normal law.

The work of Robert Gibrat resulted in a number of works devoted to applying of the law of proportional growth for various markets, industries and business sectors. In these works, the questions of fulfillment or nonfulfillment of Gibrat's Law for certain subject areas are investigated.

T. Dunne, M. Robertson, L. Samuelson, V. Hall, D. Evans analyzed the conditional density of probability distribution $p(g \mid x)$ of growth rates $g$ for firms with a characteristics vector $x$. The density of the growth rate of surviving firms $h(g \mid x)$ and the density of the measured growth rates $f(g \mid x)$ were considered separately. V. Hall and D. S. Evans [4,5] applied a technique of simultaneous assessing growth regression and mortality regression using the maximum likelihood method. T. Dunne, M.J. Robertson and L. Samuelson [6, 7] proposed a method of grouping of enterprises by intervals in accordance with their size and age. Consistent estimates of the parameters of the distribution of growth rates $h(g \mid x), f(g \mid x)$ were obtained under the assumption that enterprises within each group in terms of size and age are homogeneous with accuracy to a random component with zero mean and constant dispersion. D.S. Evans and T. Dunne, M.J. Robertson and L. Samuelson found out following statistical regularities: the probability of survival increases with the company size increasing; the growth rate of surviving firms decreases with the company size increasing; for any given firm size, the growth rate becomes smaller with increasing the size, but the probability of survival becomes greater.

Works on the survival of enterprises deal with the firm survival probability $\operatorname{Pr}$ (survival) considered as a function depending on enterprise characteristics, industry characteristics, characteristics of the environment functioning and ways of organizing the production process. D. Evans studied the relationships among firm growth, firm size, and firm age for manufacturing firms. As a basic model, D.S. Evans considered the "selective selection" model and obtained a probit model based on it. An analysis of the resulting regression equation shows that the probability of survival is positively but non-linearly affected by the size and age of the enterprise.

D.B. Audretsch [8] found out that the firm survival probability depends on the technological regime and the interindustry characteristics such as economies of scale and capital capacity. The results show that the minimum efficient level of output increasing in this sector decreases the probability of new-firm survival and the ability to achieve average branch size. If a share of innovative activity of small enterprises in the total innovation activity increases then the probability of the prevailing business regime in the industry and the survival of new enterprises increases too.

The functioning of firms is influenced by sectoral and industry specifics, and the successful life cycle of a new enterprise depends directly or indirectly on market specifics and the structure of demand.

K.V. Arshakuni $[9,10]$ fulfilled a multifactor research on genesis and development of small innovative enterprises. The author tests hypotheses about the influence of various types of state aid on the genesis and dynamics of new enterprises, as well as hypotheses about the nature of the influence of factors that determine the formation of the initial conditions for the formation of new enterprises and their subsequent evolution. In particular, these hypotheses relate to the impact of the size of initial capital on the survival and dynamics of employees in new enterprises.

Yu.S. Pinkovetskaya [11] analyzed the size distribution of small enterprises. It is proved that the number of employees is the most constructive characteristics among the various characteristics of small firms used in practice because it reflects the specificity of small enterprises and how they differ from other types of firms. The author considered mathematical models of firm size distribution for small enterprises. These models were tested to estimate small enterprises' characteristics in the Russian Federation and in particular Russian regions in 2007. The results indicate that the size distribution of small enterprises obeys a normal distribution with the density

$$
f(x)=\frac{1}{2,3 \sqrt{2 \pi}} e^{\frac{(x-8,66)^{2}}{10,54}} .
$$

This article discusses the theoretical and algorithmic aspects of the application of mathematical models based on the kinetic equation of transfer to assess the dynamics of the distribution of employment in the system of small and medium-sized enterprises in a region. The study is based on the work of A.T. Mustafin and A.K. Kantarbayeva [12], in which a mathematical model of the distribution by size of the population of firms was built and its solution to the stationary case was analyzed.

\section{METHODOLOGY AND RESUlTS}

Consider a population of small enterprises functioning in a whole region or within a particular industry. The existence of a population occurs in conditions of constant organizational changes associated with the processes of creation, growth and closure of its objects [13].

Consider a differential equation that reflects the differential conservation law for the number of objects of a certain size within the analyzed population:

$$
\frac{\partial n}{\partial t}+\frac{\partial(n \cdot r)}{\partial x}=Q(x, t)-D(x, t) \cdot n(x, t),
$$

where $n(t, x) d x$ is the number of objects having a size in the range from $x$ to $x+d x ; \quad r(t, x)=\frac{d x}{d t}$ is the speed of resizing objects of this type; $Q(x, t)$ is the number of new objects of 
this type created at time $t ; D(x, t)$ is the relative frequency of closure of objects. The equation aggregates three streams that determine the dynamics of the distribution of population objects by size: a stream of objects whose size goes beyond the interval $[x, x+d x]$; a stream of new appearing objects with a size belonging to the interval $[x, x+d x]$; stream of objects leaving the population with sizes in the interval $[x, x+d x]$.

Equation (1) can be converted with normalization to:

$$
\frac{\partial v}{\partial t}+\frac{\partial(v r)}{\partial t}=\frac{Q}{N}-\left(D+\frac{1}{N} \int Q d x-\int D v d x\right) v,
$$

where $v(x, t)=\frac{n(x, t)}{N(t)}, \quad N(t)=\int_{0}^{\infty} n(x, t) d x$ is total number of objects of the analyzed population. The function $v(x, t)$ should satisfy the following conditions:

$$
v(0, t)=v(\infty, t)=0, \quad \int_{0}^{\infty} v(x, t) d x=1 .
$$

We denote by $E(t)$ the total number of people employed at the objects of the analyzed population at the time point $t$. The value $E(t)$ can be calculated using the integral:

$$
E(t)=N(t) \int_{0}^{\infty} v(x, t) x d x,
$$

and the variation of this function satisfies the differential equation:

$$
\frac{d E}{d t}=N(t) \cdot\left(\int_{0}^{\infty} v r d x+\frac{1}{N} \int_{0}^{\infty} Q x d x-\int_{0}^{\infty} D v x d x\right)
$$

In order to solve equations (1) and (2) it is necessary to determine the initial conditions $N(0), v(0, x)$ and the functions $D(t, x), Q(t, x), r(t, x)$.

At first, we consider a stationary case, which describes the process of population development, when the main functions $N(t), \quad v(t, x), \quad D(t, x), \quad Q(t, x), \quad r(t, x)$ are clearly timeindependent. For this stationary case, the following relations hold:

$$
\begin{array}{ll}
\frac{d N}{d t}=0, & \int_{0}^{\infty} Q d x=N \int_{0}^{\infty} D v d x, \\
\frac{d E}{d t}=0, & \int_{0}^{\infty} Q x d x+N \int_{0}^{\infty} v r d x=N \int D v x d x, \\
\frac{\partial v}{\partial t}=0, & \frac{d v}{d x}+\left(\frac{d r}{d x}+D\right) \frac{v}{r}=\frac{Q}{N r} .
\end{array}
$$

In [12], an analytical form of the size distribution function $v(x)$ of population objects was obtained for a stationary situation and particular versions of functions $D(x), Q(x)$ and $r(x)$ given in Table 1 .

This article considers a more general power law for the growth rate: $r=\beta x^{\gamma},-\infty<\gamma<1$ (solutions for $\gamma=0, \gamma=1$ are presented in table $1, \quad \gamma \rightarrow-\infty$ corresponds to the trivial case of lack of growth).
If $r=\beta x^{\gamma}$, the size of the object is the function of time $x=x_{0}(1+\bar{\beta} t) \frac{1}{1-\gamma}$ and equation (2) for the stationary case has the form:

$$
\frac{d v}{d x}+\left(\beta \gamma x^{\gamma-1}+D\right) \frac{v}{\beta x^{\gamma}}=\frac{Q}{N \beta x^{\gamma}}
$$

TABLE I. ANALYTICAL SOLUTION FOR SPECIAL CASES OF STATIONARY SITUATION

\begin{tabular}{|l|c|c|c|}
\hline & $r(x)$ & $Q(x)$ & $D(x)$ \\
\hline 1 & $r=$ const & \multirow{2}{*}{$Q(x)=\frac{Q_{0}}{\lambda} \cdot \exp \left(-\frac{x}{\lambda}\right)$} & $D=$ const \\
\hline 2 & $r=\beta x$ & $\frac{v(x)}{2}(x)=\frac{\exp (-x / \tilde{x})-\exp (-x / \lambda)}{\tilde{x}-\lambda}$ \\
\hline 1 & \multicolumn{2}{|c|}{$v(x)=\frac{D / \beta}{x_{*}}\left(\frac{x}{x_{*}}\right)^{-1-\delta}, x \geq x_{*}>0$} \\
\hline 2
\end{tabular}

In order to solve equation (7), we use the numerical Euler's method. Taking into account the singularity of the equation, we introduce the lower restriction on the size range $x \geq x^{*}>0$, and the upper restriction $x \leq B<\infty$.

To implement the Euler's method [14], the following difference scheme is used in the work:

$$
\begin{gathered}
v_{i+1}=v_{i}+\left(\frac{\delta}{\lambda} \frac{\exp \left(-\frac{x}{\lambda}\right)}{x_{i}^{\gamma}}-\left(\gamma+\frac{\delta}{x_{i}^{\gamma-1}}\right) \cdot \frac{v_{i}}{x_{i}}\right) \cdot \Delta x_{i}, \delta=\frac{D}{\beta}, \\
\sum_{i=0}^{M} v_{i} \cdot \Delta x_{i}=1 .
\end{gathered}
$$

The accuracy of the calculations is provided by the choice of the step $\Delta x_{i}$

Figure 1 shows the results of a numerical solution for various values of the power dependence of the growth rate (Fig. 1).

The analysis of numerical calculations shows that when the power of the function $r=\beta x^{\gamma}$ the distribution shifts $v(x)$ to the region of small objects.

A variation in the power of the growth function $r=\beta x^{\gamma}$ provides an opportunity to vary the distribution $v(x)$ in wide range. This allows to a certain extent solving the inverse problem. Based on the experimental distribution $v(x)$ obtained from statistical data processing, we will be able to restore the growth function for further solving the problem of forecasting the movement of labor resources and ensuring optimal employment. 


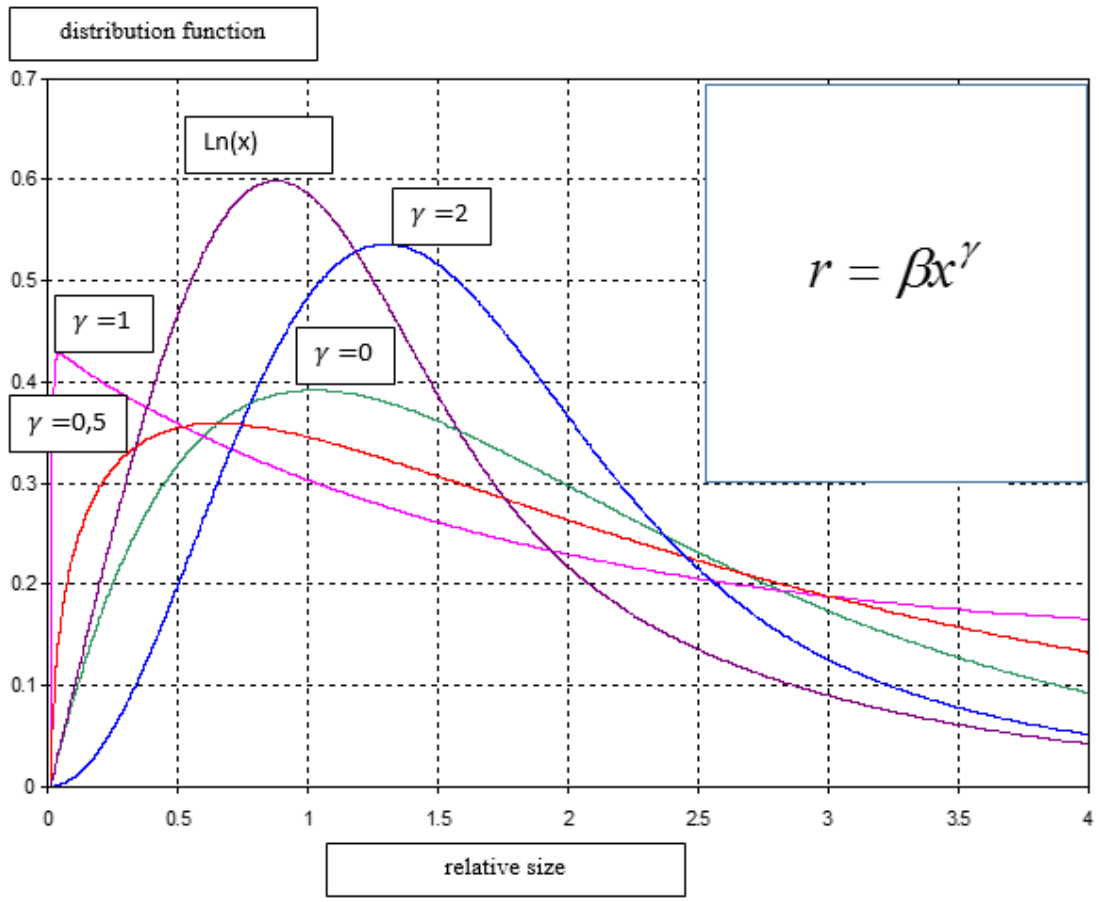

Fig. 1. Employment distribution function for various growth rate laws

The above calculations have been carried out for the function $D=$ const, however, a numerical solution allows using other types of functions, for example, functions that reflect the assumption that the probability of closure decreases with increasing numbers. Choosing functional dependencies, it is necessary to consider that

$$
N=\frac{\int_{0}^{\infty} Q d x}{\int_{0}^{\infty} D v d x} .
$$

If $N$ is finite, the function $D(x)$ must satisfy the condition:

$$
0<\int_{0}^{\infty} D \cdot v d x<\infty
$$

This condition holds for the decreasing exponential and the power law

$$
\begin{gathered}
D(x)=D_{0} \cdot e^{-\mu x}, \\
D(x)=\left\{\begin{array}{l}
D_{0}, \quad 0 \leq x \leq x^{*} \\
D_{0} \cdot\left(\frac{x}{x^{*}}\right)^{\mu}, \quad x \geq x^{*}
\end{array}\right.
\end{gathered}
$$

For the existence of a solution under the power law, an additional restriction is introduced on the growth of the closing frequency at $x \rightarrow 0$.

Figure 2 shows a graphical demonstration of variation of the solutions to the differential equation under consideration for various laws of closing frequency.
The analysis of the calculation results shows that a decreasing nature $D(x)$ leads to a shift of the function $v(x)$ towards objects with a large number of employed.

In this paper we also investigate a non-stationary case, when the behavior of the functions $D(t, x), Q(t, x), r(t, x)$ depends explicitly on time. A numerical solution of equation (2) is used whenever the integral condition (3) is satisfied. For the numerical solution of the equation, we apply the «finite volume» method developed for technical applications [14]. Let us briefly discuss the essence of this method. The range of variable $x$ is divided into a finite number of intervals, the socalled "finite volumes". Values depending on $x$ are replaced by an average value on each of the intervals. Separate volumes are connected with equations of balance of determining quantities, and we get a system of ordinary differential equations.

The obtained system is solved by numerical methods adapted for large dimensions. Consider this method for solving equation (1). The domain of definition is divided into I intervals, a certain large number $X^{*}$ is taken as the right border. Equation (1) is transformed into a system of ordinary differential equations:

$$
\frac{d n_{i}}{d t}=n_{i-1} \cdot r_{i-1}-n_{i} \cdot r_{i}+Q_{i}-D_{i} \cdot n_{i}, i=1 \ldots . . I
$$

where $n_{i}=\int_{x_{i-1}}^{x_{i}} n(x) d x, r_{i}=r\left(x_{i}\right), Q_{i}=\int_{x_{i-1}}^{x_{i}} Q(x) d x, D_{i}=D\left(\tilde{x}_{i}\right)$.

Suppose that at the initial moment of time the distribution of objects by size $n_{i}=$ const $, \forall i, n_{0}=0$. 


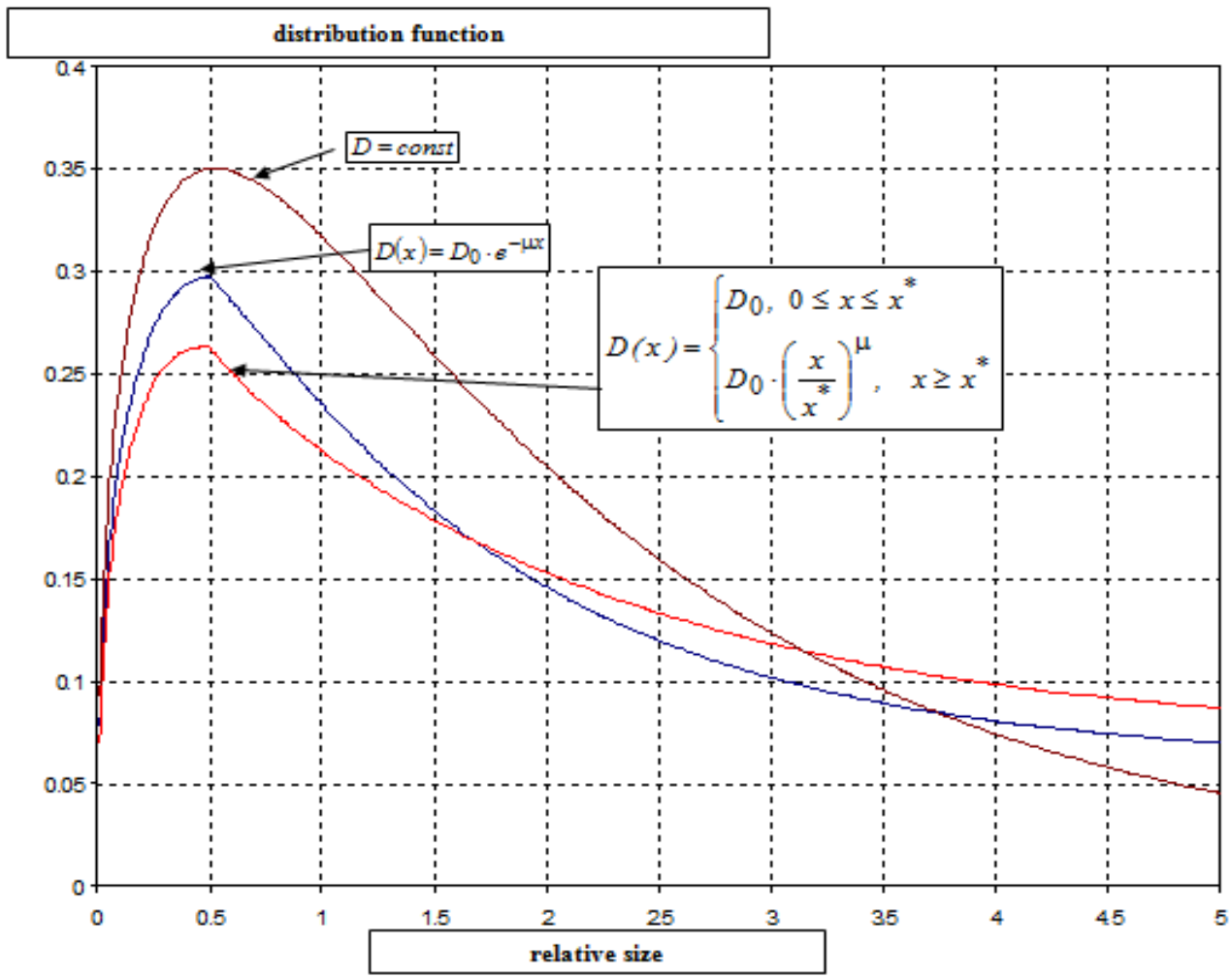

Fig. 2. Solutions of differential equation (2) for various types of functions of the relative frequency of closure of population objects

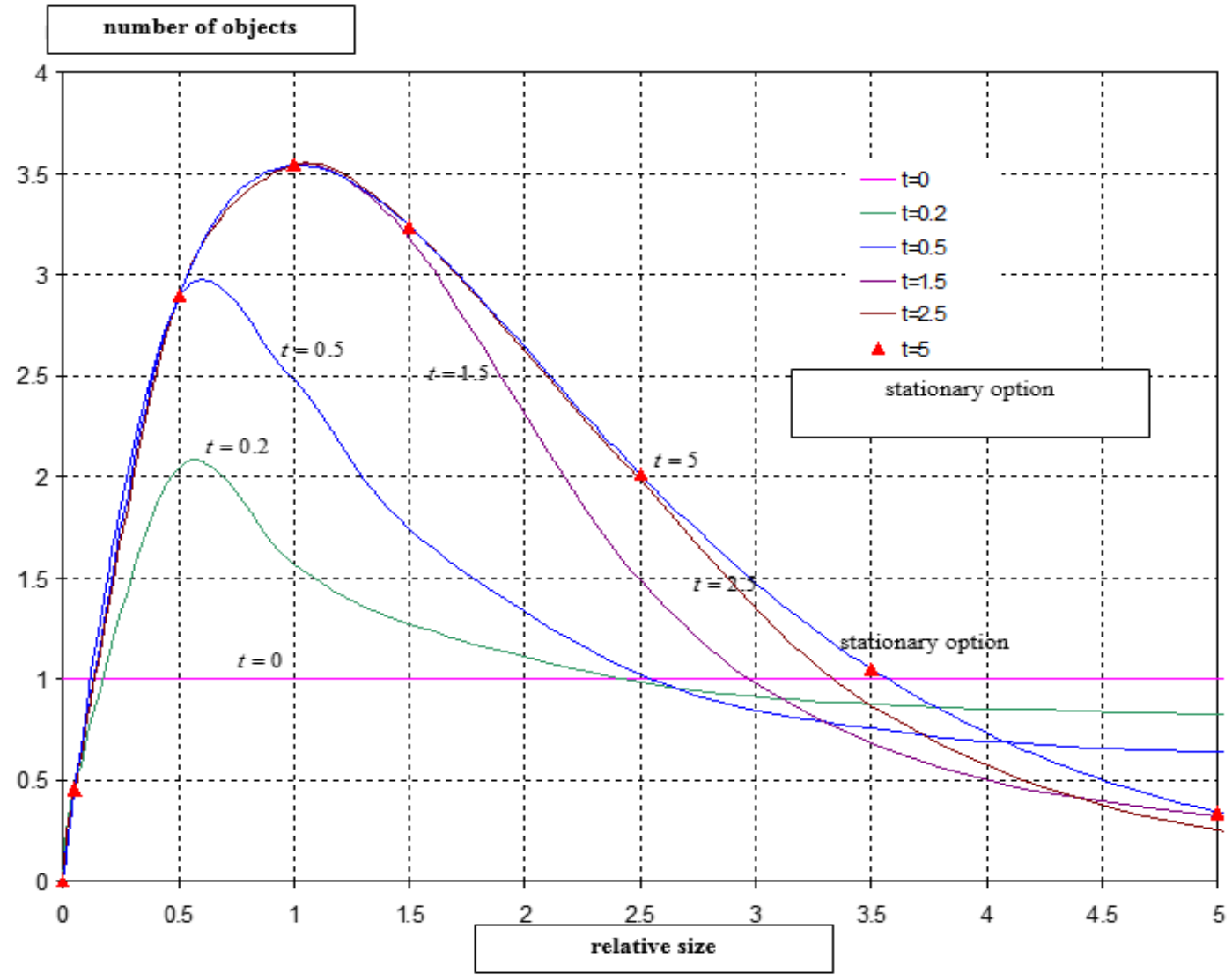

Fig. 3. The results of numerical calculations for the non-stationary case (by periods) 


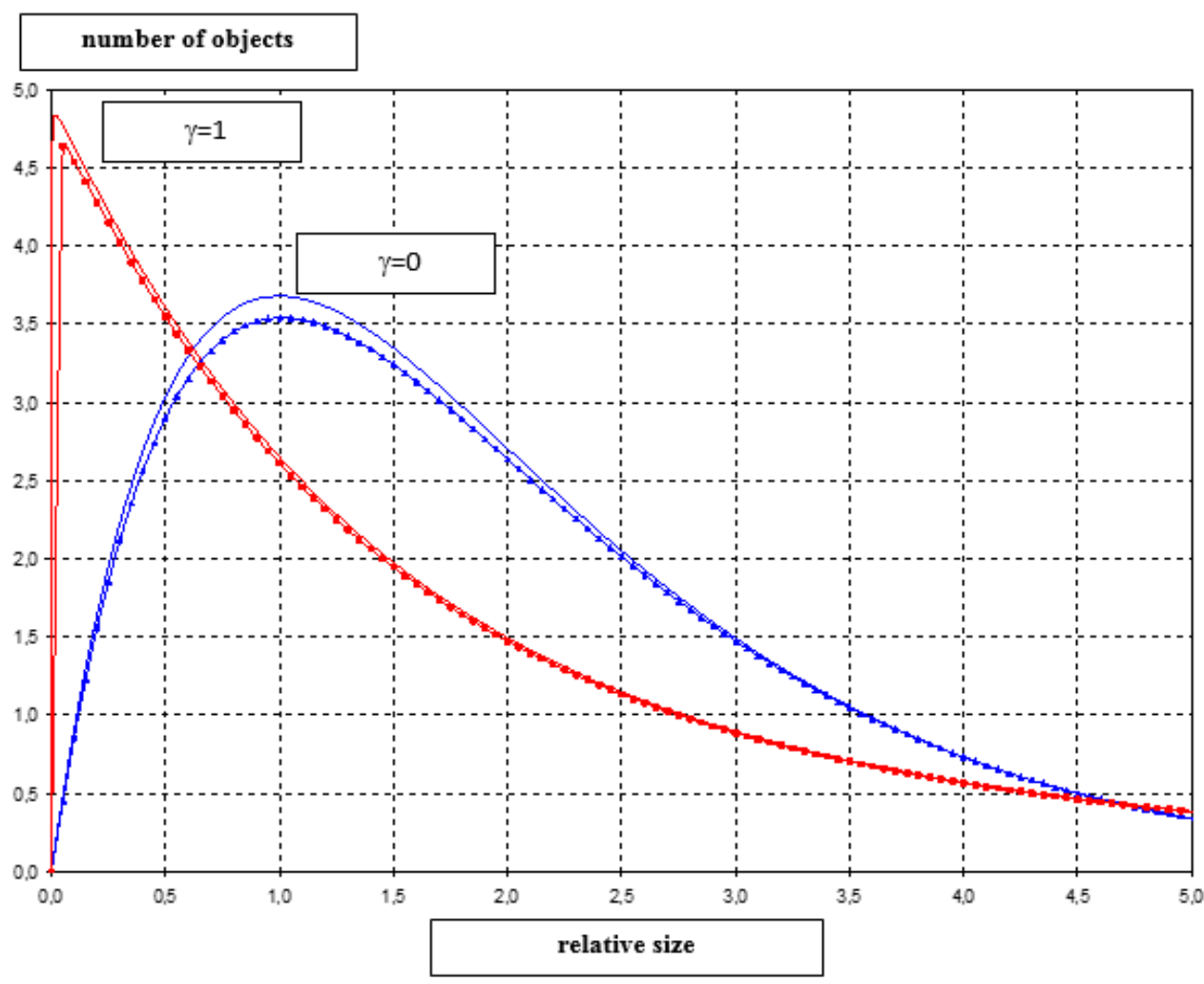

Fig. 4. Comparison of solutions of stationary and non-stationary equations

For the numerical solution of the resulting system, we use the Euler's method:

$$
n_{i}^{k+1}=n_{i}^{k}+\left(\left(n_{i-1}^{k} \cdot r_{i-1}-n_{i}^{k} \cdot r_{i}\right) / \Delta x_{i}+Q_{i}-D_{i} \cdot n_{i}^{k}\right) \cdot \Delta t_{k},
$$

where $i=1 \ldots I$ is the number of intervals for partitioning a domain $X, \Delta x_{i}$ is the interval size; $k=1 . . . K$ is the number of time steps, $\Delta t_{k}$ is the time step.

The accuracy of the solution is determined by the choice of the time step $\Delta t_{k}$ and the step of partitioning the domain $x$. The results of the numerical solution are shown in Figure 3. The function $v(x, t)$ deforms rather quickly and approaches the obtained stationary solution with the functions $D(x)$, $Q(x), r(x)$ after 5 periods.

The solution of the non-stationary problem allows, with the adopted functional dependencies, to assess the dynamics of employment $E(t)$ in small innovative enterprises (Fig. 5).

The distribution $v(x, t)$ and through it the employment $E(t)$ in the considered population of enterprises can be influenced by choosing the type and parameters of functions $D(t, x), Q(t, x), r(t, x)$. At the beginning of the article, we gave an overview of the works devoted to the analysis of the considered functional dependencies, and highlighted a number of significant factors that influence their formation. It is possible to regulate the process of forming these functions purposefully from the position of the management center in order to achieve the goals of population development.

We introduce the following quantities: $\Phi(x, t)$ is the cost of creating a new size object $x ; \Psi(x, t)$ is the released value when closing an object of size $x ; h(x, t)$ is the cost of creating one job in the process of object growth.

Based on the introduced functions, it is possible to calculate the cost of the processes of creation, growth and closure of population objects:

$$
C_{s}(t)=\int_{0}^{\infty}(\Phi(x, t) Q(x, t)+h(x, t) r(x, t) n(x, t)-D(x, t) \Psi(x, t) n(x, t)) \cdot d x
$$

It is assumed that the cost for any time is limited from above $C_{s}(t) \leq C_{0}(t)$.

As target criteria for regulating the processes of creation, growth and closure of population objects, one can consider maximizing the total number of workers employed at population objects:

$$
E(t)=\int_{0}^{\infty} n(x, t) x d x \rightarrow \max
$$

and (or) minimizing the costs of process regulation:

$C_{s}(t)=\int_{0}^{\infty}(\Phi(x, t) Q(x, t)+h(x, t) r(x, t) n(x, t)-D(x, t) \Psi(x, t) n(x, t)) d x \rightarrow \min$

The stationary maximization problem $E(t)$ takes the form: 


$$
\begin{gathered}
r \frac{\partial n}{\partial x}+n \frac{\partial r}{\partial x}=Q(x)-D(x) \cdot n \\
\int_{0}^{\infty}(\Phi(x) Q(x)+h(x) r(x) n(x)-D(x) \Psi(x) n(x)) \cdot d x \leq C_{0}, \\
\int_{0}^{\infty} n(x) \cdot x \cdot d x \rightarrow \max
\end{gathered}
$$

Consider a cost function that satisfies the following conditions: there is a constant cost component that does not depend on the size of the objects; unit costs $\phi(x)$ per worker initially decrease, then increase, and then stabilize with increasing size:

$$
\phi(x)=A_{1}-B_{1} \cdot e^{-\alpha(x-a)^{2}}+B_{2} \cdot e^{-\beta \cdot x} .
$$

Consider the costs of creating new jobs in the growth process identical to the unit costs in the event of an economic entity.

As a function characterizing the released value with the closure of entities, we consider the function

$$
\Psi(x)=A_{3}+\sqrt{x} \cdot\left(A_{4}+B_{3} \cdot e^{-\delta \cdot(x-b)^{2}}\right) .
$$

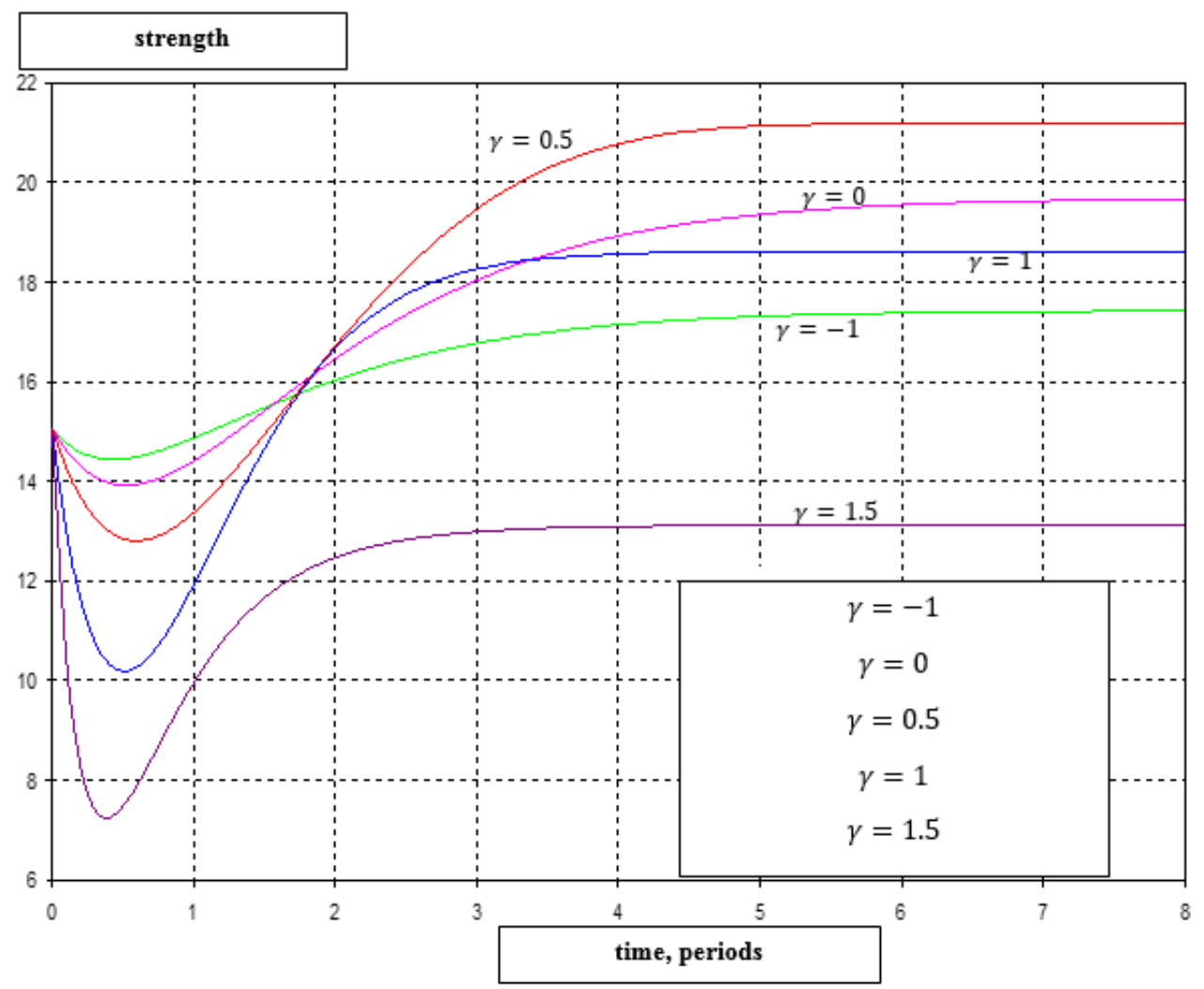

Fig. 5. Employment Dynamics $E(t)$

In order to solve problem (13), we used the simulation method of choosing the optimal solution on a certain set of alternatives.

\section{CONCLUSION}

The tools applied to study the dynamics of the population of enterprises analyzed in the work allow obtaining the distribution of population objects by size depending on the type of functions of their life cycle, creation and closure; determine the number of people working at population objects and analyze the effectiveness of various strategies for regulating the processes of creation, closure and growth of population objects. The solution of these problems on the basis of high-quality regional statistics will allow formulating a system of recommendations for regulating at the regional or sectoral level the processes of support and future development of employment in the context of organizational changes in the digital economy.

\section{Acknowledgment}

The reported study was funded by the Russian Foundation for Basic Research according to the research project No. 1929-07400 mk.

\section{References}

[1] Y.A. Salikov, I.V. Logunova, I.V. Kablashova, "Trends in human resource management in the digital economy", Proc. of the Voronezh State Univer. of Engineer. Technol., vol. 81, no. 2, pp. 393-399, 2019.

[2] N.A. Serebryakova, I.V. Avdeev, "The content of structural transformations of the region's economy, adequate to the requirements of digitalization", Proc. of the Voronezh State Univer. of Engineer. Technol., vol. 80, no. 4, pp. 408-412, 2018.

[3] R. Gibrat, Les inégalités économiques; applications : aux inégalités des richesses, à la concentration des entreprises, aux populations des villes, 
aux statistiques des familles, etc., d'une loi nouvelle, la loi de l'effet proportionnel. Paris: Librairie du Recueil Sirey, 1931.

[4] D.S. Evans, "Tests of Alternative Theories of Firm Growth", J. of Political Econ., vol. 95, no. 4, pp. 657-674, 1987.

[5] B.H. Hall, "The Relationship between Firm Size and Firm Growth in the U.S. Manufacturing Sector”. J. of Industr. Econ., vol. 35, no. 4, pp. 583605, 1987.

[6] T. Dunne, M.J. Roberts, L. Samuelson, "Patterns of Firm Entry and Exit in U.S. Manufacturing Industries", Rand J. of Econ, vol. 19, pp. 495-515, 1988.

[7] T. Dunne, M.J. Roberts, L. Samuelson, "The growth and Failure of U.S. Manufacturing Plants”, Quarterly J. of Econ., vol. 104, no. 4, pp. 671-698, 1989.

[8] D.B. Audretsch, "New-Firm survival and Technological Regime", Rev. of Econ. and Statist., vol. 73, no. 3, pp. 441-450, 1991.

[9] K.V. Arshakuni, "The processes of emergence and formation of new small enterprises. Econometric study of the initial conditions of genesis based on individual data", HSE J. of Econ., vol. 9, no. 1, pp. 17-50, 2005 .

[10] K.V. Arshakuni, "The dynamics of new small enterprises and endogenous initial conditions. Simulated Likelihood Econometric Approach", HSE J. of Econ., vol. 9, no. 3, pp. 291-324, 2005.

[11] Yu.S. Pinkovetskaya, "The number of small businesses: analysis results”, J. Reg. Econ., vol. 2, no. 18, pp. 224-230, 2009.

[12] A.T. Mustafin, AK. Kantarbayeva, "On the distribution of firms by size", Econ. and mathem. Methods, vol. 3, pp. 105-112, 2000.

[13] N.B. Baeva, "Cembartsev DS."Employment forecasting in the regional economic system based on the regulatory and statistical approach", vol. 1, pp. 180-186, 2006 [Mater. of the Int. Sc. and Pract. Conf. Economic Forecasting: Models and Methods].

[14] V.E. Miln, Numerical solution of differential equations. Moscow: Izdatel'stvo inostrannoy literatury, 1955. 